\title{
Das Ziel ist Gesellschaftsfähigkeit
}

\section{Anmerkungen zu den Aufgaben der Jugendsozialarbeit}

\author{
口 Joachim König
}

Jugendsozialarbeit ist Teil der Jugendhilfe mit einem klaren Erziehungsauftrag für eine bestimmte Zielgruppe. Auf diesem pädagogischen Mandat aufbauend, hat sie jedoch auch einen Bildungsauftrag, der über die Qualifizierung und Wissensvermittlung hinausgeht.

1.

Dieser Beitrag versucht Klarheit über den Bildungsauftrag der Jugendsozialarbeit herzustellen und darauf aufbauend - vor dem Hintergrund eines dafür entwickelten Begriffs von Bildung - zur Standortbestimmung und Profilbildung innerhalb der Jugendsozialarbeit beizutragen. Dazu wird versucht, zunächst aus drei Richtungen,

- einer soziologischen, den Zustand unserer Gesellschaft in den Blick nehmend,

- einer juristischen, die rechtlichen Regelungen betrachtend,

- und einer inhaltlich-konzeptionellen, die Praxis der Jugendsozialarbeit näher analysierend

auf dieses Arbeitsfeld zu blicken, dabei den Bedarf von Bildung als Ziel und Methode für die Praxis zu klären. Darauf aufbauend wird innerhalb des Feldes der Begriff von Bildung inhaltlich vertieft und für die Praxis konkretisiert.

\section{2.}

Prof. Dr. Joachim König lehrt an der Evangelischen Fachhochschule Nürnberg im Fachbereich Sozialwesen und ist Leiter der dortigen Arbeitsstelle für Praxisforschung und Evaluation im kirchlichen, sozialen und Bildungsbereich.

E-Mail

joachim.koenig@efh-nuernberg.de formel geworden. Auch die Vielzahl der Lebensformen, der biografischen Möglichkeiten, der geografischen Perspektiven ist ständig im Wachstum und - das macht die Sache noch schwieriger - in einem ständig beschleunigten Wandel begriffen. Es gibt auf dieser Welt nichts mehr, was es nicht gibt, alles ist möglich, jedem Menschen steht, so scheint es zumindest, alles offen. Jeder könne machen was er will, so die zentrale Illusion. Gleichzeitig fallen die ethischen und moralischen Schranken, welche eine Begrenzung dieser Freiheiten bedeuten könnten, zusehends. Und auch die technischen Möglichkeiten wachsen, um dieser Freizügigkeit (wie es eigentlich heißen müsste) immer mehr Türen und Tore zu öffnen. Die zweite große Freisetzung des Individuums seit der Aufklärung, wie es Ulrich Beck einmal formuliert hat, ist in vollem Gange.

Das klingt - zugegeben - in gewisser Weise verlockend, auch für uns als pädagogisch Verantwortliche, zumal ja auch die pädagogischen Bemühungen immer davon leben, Selbstbestimmung und damit das Ende der Bevormundung, Einschränkung und Lenkung zu erreichen. Doch: Der große Haken zeigt sich spätestens beim zweiten Begriff, mit dem diese postmoderne Gesellschaft beschrieben wird, nämlich dem der Individualisierung. Freisetzung aus Vorgegebenem, Befreiung von Einschränkungen und Regeln, Normen und Festlegungen bedeutet immer einen Mehraufwand an individueller Entscheidung und Verantwortlichkeit. Und diese Aufgabe wird individualisiert, also dem Einzelnen überlassen: Jeder kann machen was er will, aber bitteschön: Er oder sie muss auch die Verantwortung dafür übernehmen, dass er sich so und nicht anders entschieden hat. Und auch die Frage nach den Ressourcen, die notwendig sind im Hinblick auf die Konsequenzen einer Entscheidung, fällt zurück auf das Individuum. An dieser Stelle wird der Freiheitsbegriff, der hinter 
einer solchen Grundannahme steckt, in vielerlei Hinsicht problematisch. Im Grunde geht es eigentlich nicht um eine "Freiheit $\mathrm{zu}$ ", sondern nur um die schlichte »Freisetzung aus « - aus Verbindlichkeiten, Verlässlichkeiten und aus im Grunde positiv zu beurteilenden $\mathrm{Zu}$ sammenhängen.

Was will ich werden? Was will ich sein? Was soll aus mir werden? Das sind die drängenden Fragen, gerade im Jugendalter. Und diese Fragen dürfen Jugendliche selber entscheiden, aber sie müssen sie auch selber entscheiden in dieser offenen, immer komplexeren, unübersichtlicheren und immer vieldeutigeren Gesellschaft. Jugendliche dürfen selber entscheiden und - oder besser aber - sie müssen sich vor allem selber entscheiden können!

Was sind aber die Kriterien, die Orientierungen, die Entscheidungsgrundlagen und Entscheidungshilfen? Das ist der Punkt, an dem vor allem Jugendliche, mit ihren drängenden Fragen nach Identität, nach persönlicher, beruflicher und sozialer Zukunft und Unverwechselbarkeit nicht nur enorm gefordert, sondern oft überfordert sind. Jugendliche mit schlechten Startchancen erleben dieses Dilemma »knallhart individualisiert «: Alles scheint möglich auf dieser Welt, die Verheißun- gen der Märkte im medialen Hochglanz sind riesig. Aber: Was will ich? Wonach soll ich mich entscheiden? Kann ich das dann überhaupt? Was hat welche Konsequenzen? Fragen, die vor allem nach einem verlangen: nach mehr Klarheit und Übersichtlichkeit, nach mehr Eindeutigkeit, Struktur und Verlässlichkeit.

Und an diesem Punkt ist Bildung gefragt, gerade in der Jugendsozialarbeit, gerade in der Arbeit mit benachteiligten Jugendlichen (und eben nicht in erster Linie an Gymnasien, Hochschulen und Volkshochschulen). Bildung als Ziel und als Methode zur Herstellung von mehr Orientierung, mehr Eindeutigkeit und Durchschaubarkeit in dieser Welt, in der - vor allem aus der Sicht der Betroffenen - die Maßstäbe immer mehr verloren gehen, Widersprüche und Ambivalenzen sich hingegen häufen und - seien wir ehrlich - niemand mehr einen wirklich vollständigen Überblick hat.

Erstes Fazit also, mit Blick auf die Gesellschaft: Bildung kann als eine Möglichkeit betrachtet werden, methodisch und perspektivisch Orientierungen in der Uneindeutigkeit zu bieten, in zunehmender Komplexität für mehr Klarheit zu sorgen, gerade im Hinblick auf die Persönlichkeitsentwicklung von benachteiligten Jugendlichen.
3.

Jetzt zur rechtlichen Perspektive: Nach Paragraf 13 Absatz 1 des Kinder- und Jugendhilfegesetzes (SGB VIII) legt das Zentrum der Jugendsozialarbeit fest: »Jungen Menschen, die zum Ausgleich sozialer Benachteiligungen oder zur Überwindung individueller Beeinträchtigungen in erhöhtem Maße auf Unterstützung angewiesen sind, sollen im Rahmen der Jugendhilfe sozialpädagogische Hilfen angeboten werden, die ihre schulische und berufliche Ausbildung, Eingliederung in die Arbeitswelt und ihre soziale Integration fördern.«

Dies ist zwar eine klare, aber sehr allgemein formulierte Bestimmung, die zur Klärung des Standortes der Jugendsozialarbeit, zur Identifizierung des spezifischen Auftrages zwar schon in eine bestimmte Richtung weist:

- »in erhöhtem Maße auf Unterstützung angewiesen «

- »sozialpädagogische Hilfen sollen geleistet werden «

- »Das Ziel ist die berufliche und die soziale Integration."

Dies reicht aber zur Klärung eben noch nicht aus, denn erstens können sich da fast alle wiederfinden, die irgendwann schon einmal pädagogisch oder sozialpä-

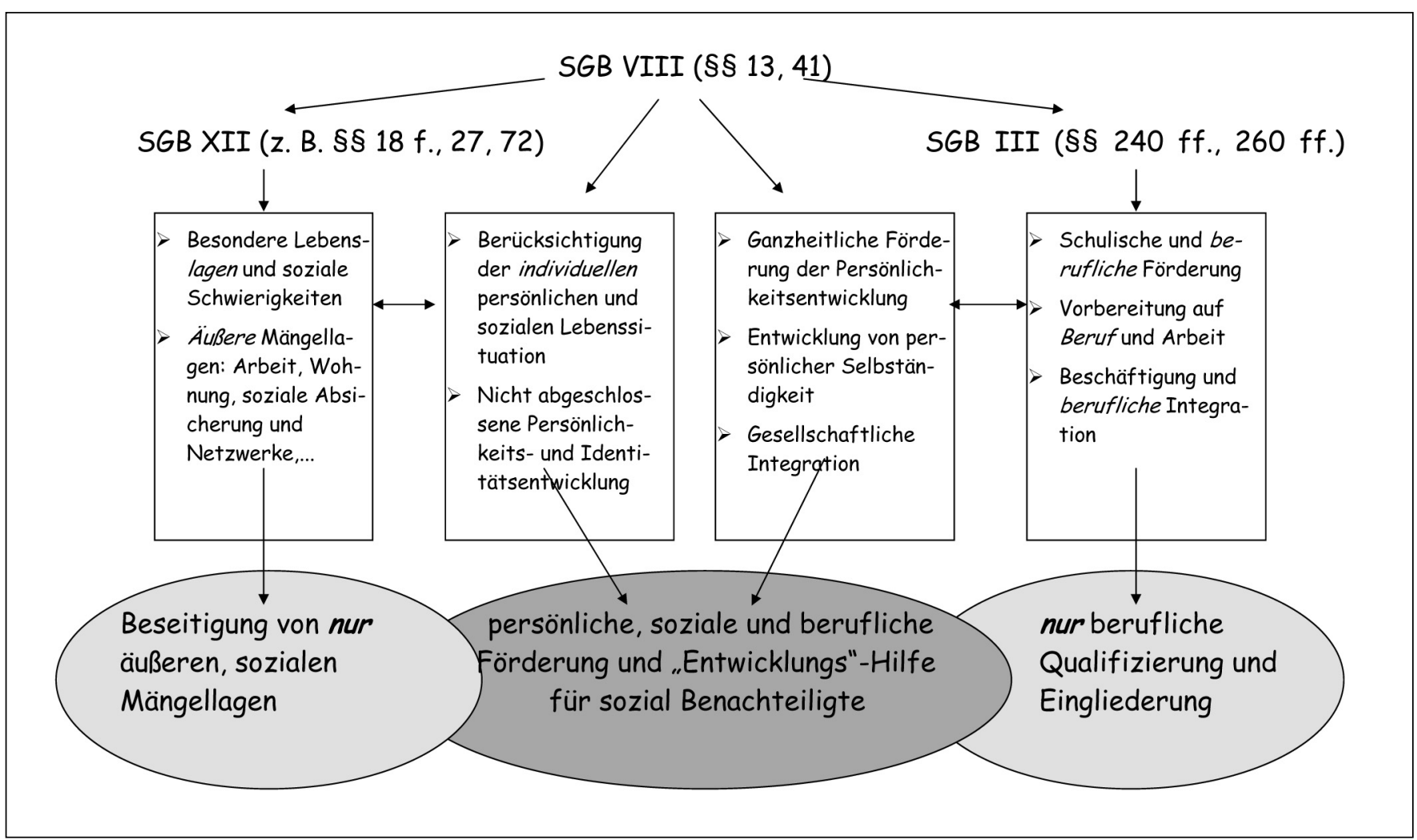

Abb. 1: Das Zentrum der Jugendsozialarbeit? - Rechtliche Abgrenzung 
dagogisch mit Jugendlichen gearbeitet haben. Was heißt denn schon »erhöhter Förderbedarf «, „sozialpädagogisch «, »Eingliederung fördern «. Und zweitens stehen neben dem Kinder- und Jugendhilfegesetz auch in anderen Gesetzestexten ähnliche Passagen, die in diese Richtung gehen und so zu Verwechslungen, zu Unschärfen im Profil führen können.

Da ist erstens das Gesetz zur Rehabilitation und Teilhabe behinderter Menschen (SGB IX), wo es zwar im Wesentlichen um behinderte Menschen, aber eben auch um Menschen geht, die von Behinderung bedroht sind. Da ist auch die Rede von Leistungen zur Integration in das Arbeitsleben ( $\$ 33 \mathrm{ff}$.) im Sinne von beruflicher Eingliederung und von Leistungen zur Integration in das Leben in der Gemeinschaft ( $\mathbb{S} 55 \mathrm{ff}$.) im Sinne sozialer Eingliederung.

Da ist zweitens das Arbeitsförderungsgesetz (SGB III), wo es um die sozialpädagogische Begleitung der Berufsausbildung von benachteiligten Jugendlichen in den ausbildungsbegleitenden Hilfen geht oder um die Förderung der beruflichen Ausbildung von benachteiligten und behinderten jungen Menschen in überbetrieblichen Einrichtungen ( $\$ 240 \mathrm{ff}$.) oder um die berufliche Eingliederung von Langzeitarbeitslosen geht, etwa durch deren Beschäftigung in Arbeitsbeschaffungsmaßnahmen (\$ 260 ff.).
$\mathrm{Da}$ ist drittens das Sozialhilfegesetz (SGB XII), wo es um Hilfen zur Arbeit und um die Schaffung von Arbeitsgelegenheiten besonders auch für junge Menschen ( $\$ 18$ und 19), oder um Hilfen in besonderen Lebenslagen und in besonderen sozialen Schwierigkeiten zur Sicherung der Lebensgrundlage geht ( $\$ 27$ ff.).

Und da sind viertens - quer zu diesen Gesetzen - die Bestimmungen des Europäischen Sozialfonds. Dort geht es gezielt darum, benachteiligte Menschen - und eben auch Jugendliche - durch bestimmte Programme beruflich und sozial zu integrieren; allerdings hier immer verbunden mit einer europäischen Perspektive und der Voraussetzung, dass alle Maßnahmen national, also über eines der erwähnten Bundesgesetze kofinanziert sind.

Trotz dieser Überschneidungen und Unschärfen gibt es aber zwei klare Trennlinien zwischen dem Paragrafen 13 im Kinder- und Jugendhilfegesetz zur Jugendsozialarbeit und den benachbarten Gesetzen: Bei der Arbeitsförderung geht es "nur « um berufliche Qualifizierung und Eingliederung, bei der Sozialhilfe »nur « um die Beseitigung äußerer Notlagen. Im Zentrum des Auftrags der Jugendsozialarbeit als originärer Träger der Jugendhilfe muss jedoch stehen: die persönliche, soziale und berufliche Förderung sozial benachteiligter junger Men- schen (vgl. Abb. 1). Und darauf sollten wir uns, unabhängig von der Frage, wie einzelne Teile unserer Arbeit finanzierbar sind, im Wesentlichen konzentrieren. Der Übersichtlichkeit halber ist in der Abbildung nur die Abgrenzung gegenüber der Sozialhilfe und der Arbeitsförderung vorgenommen worden. Gegenüber der Behindertenhilfe ist die Trennlinie über die Zielgruppe der behinderten Menschen relativ klar und eindeutig formuliert.

Unabhängig von der Frage nach der Finanzierung der Einrichtungen scheint in der Jugendsozialarbeit also hier das eigentliche pädagogische und vor allem sozialpädagogische Zentrum zu liegen. Im Gegensatz zu

- der einseitig sozialpolitischen Ausrichtung auf der einen (SGB XII) und

- der bildungs- und arbeitsmarktpolitischen Ausrichtung (SGB III) auf der anderen Seite.

Der Kern des Anliegens der Jugendhilfe, der da zum Ausdruck kommt, kann beschrieben werden mit dem Anspruch "persönliche, soziale und berufliche Förderung und `Entwicklungshilfe für sozial benachteiligte junge Menschen « zu leisten. Und genau das sind die Formulierungen, mit denen seit mehr als 200 Jahren, seit der Zeit der europäischen Aufklärung (und immer wieder hoch aktuell) genau das formuliert wird, was mit Bil-

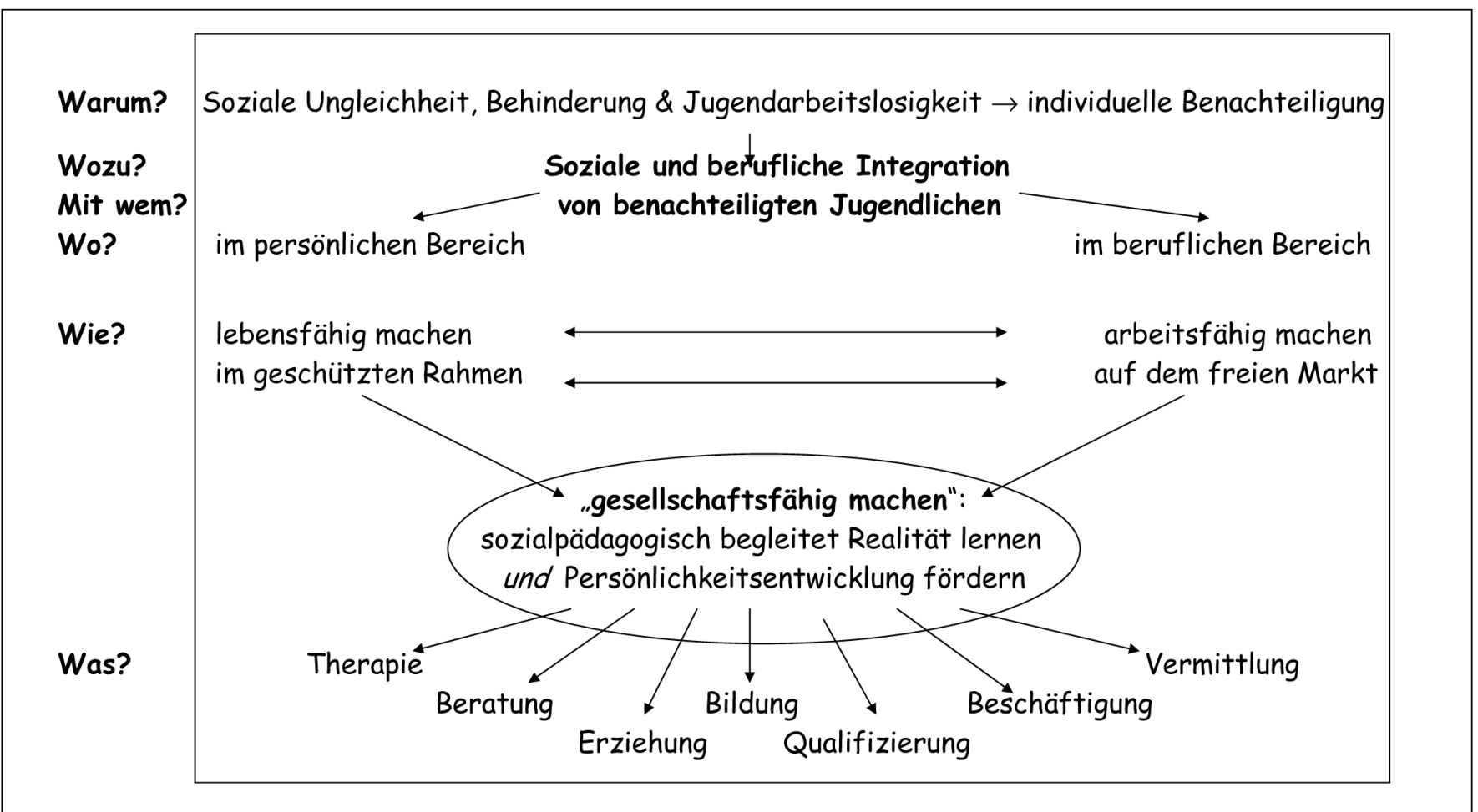

Abb. 2: Das Zentrum der Jugendsozialarbeit? - Konzeptionelle Abgrenzung 
dung gemeint ist: Persönlichkeitsentwicklung, ganzheitliche Förderung der Person und damit alle Lebensbereiche betreffend, Selbstständigkeit fordernd und gleichzeitig fördernd und - wie Humboldt das einmal genannt hat - verbunden mit dem Anspruch der Universalität, der Totalität und der Individualität.

Deshalb zweites Fazit: Richtig verstandene Bildung kann auch mit Blick auf die rechtlichen Bestimmungen im konzeptionellen Zentrum der Jugendsozialarbeit stehen.

4.

Um diese Kernaufgabe genauer und differenzierter bestimmen zu können, wenden wir uns dem dritten Annähe- rungsversuch zu. Die Frage ist jetzt: Was passiert inhaltlich, konzeptionell in der Praxis, im Alltagsgeschäft der Jugendsozialarbeit? Dazu habe ich versucht, mir einen Überblick in der Literatur und in den Konzepten und Konzeptionen zu verschaffen. An diese Texte habe ich sechs W-Fragen gestellt (vgl. Abb. 2) und so versucht, einen Überblick über das Wesentliche $\mathrm{zu}$ erreichen. Daraus ist eine

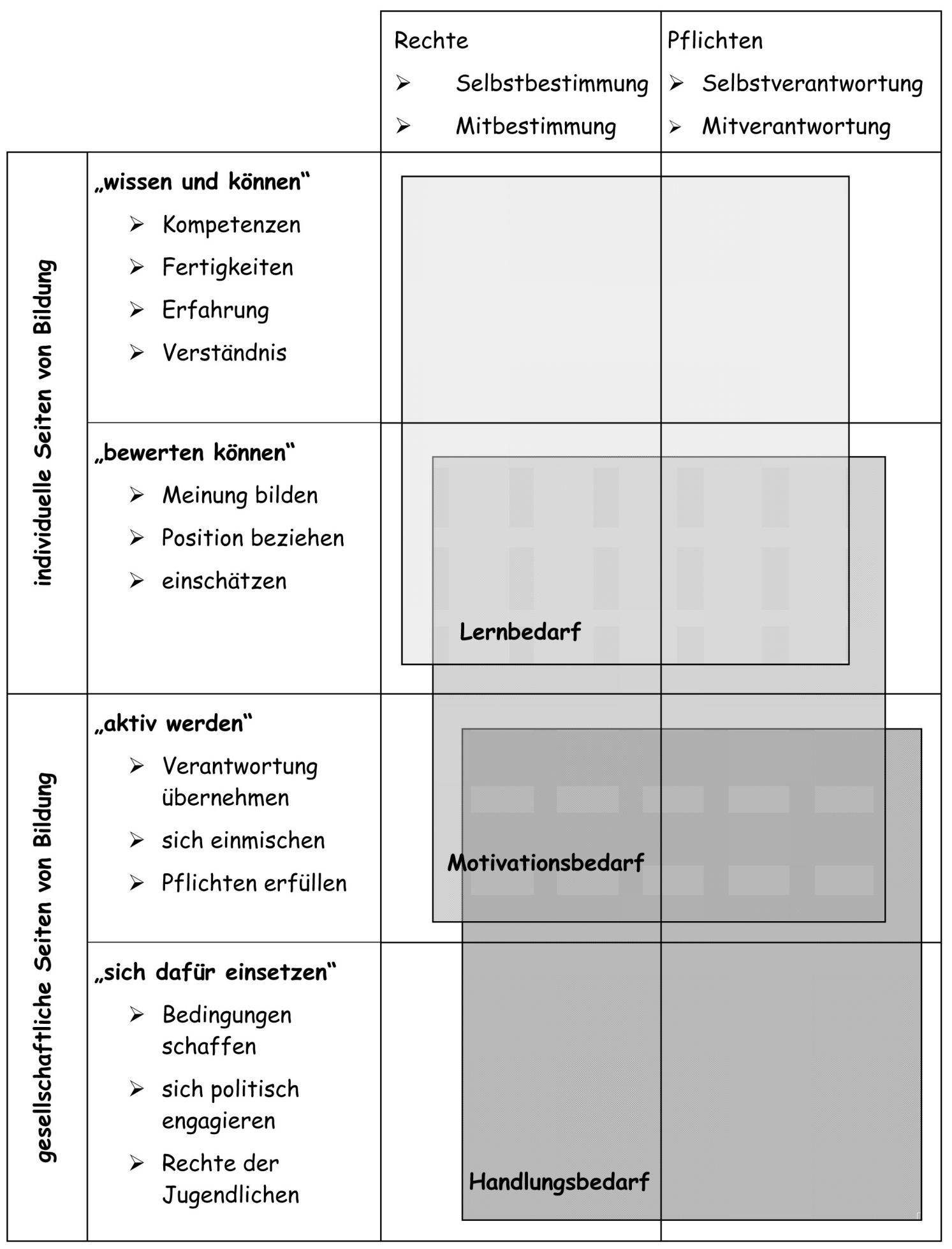

Abb. 3: Ein Praxismodell für Bildung in der Jugendsozialarbeit 
»Landkarte « entstanden, die in der Jugendsozialarbeit zur Orientierung und zur Standortbestimmung dienen kann: In was für einem Gelände bewegen wir uns? Von wo aus agieren wir? Wo ist unser Zentrum, etwa im Unterschied zu den Bildungsträgern oder zu den Werkstätten für Behinderte, aber auch im Unterschied zu den stationären Trägern der Hilfen zur Erziehung, etwa im Heimbereich?

Jugendsozialarbeit begründet sich aus der Tatsache sozialer Ungleichheit und existierender Jugendarbeitslosigkeit, die beide zu individuellen Benachteiligungen führen. Jugendsozialarbeit versucht deshalb, das Ziel sozialer und beruflicher Integration bei benachteiligten Jugendlichen zu verfolgen. Dabei geht sie zweigleisig vor. Sie setzt im persönlichen Bereich an, sucht einen geschützten Rahmen zu schaffen, um Jugendliche lebensfähig zu machen. Und sie setzt im beruflichen Bereich an, versucht Jugendliche im »freien Markt « mit der sozialen Wirklichkeit zu konfrontieren und sie arbeitsfähig zu machen. Diese Zweigleisigkeit drückt sich beispielsweise aus im Prinzip der Jugendwerkstatt, das sozialpädagogisch begleitete Realität vermittelt und dadurch Persönlichkeitsentwicklung im pädagogischen Sinne fördert. Dabei ist es notwendig im konzeptionellen Bereich alle sieben inhaltlichen Ansätze zu verfolgen: Therapie, Beratung, Erziehung, Bildung, Qualifizierung, Beschäftigung und Vermittlung.

So wird deutlich, dass Jugendsozialarbeit wirklich Aufgaben zu erfüllen in der Lage ist, die sonst einerseits von der klassischen Jugendhilfe, den Hilfen zur Erziehung, erbracht werden und andererseits von den Bildungsträgern getrennt wahrgenommen werden. Der sinnvolle Grundgedanke dabei ist: Beide Seiten, das »Lebensfähig machen « und das "Arbeitsfähig machen « von Jugendlichen, gehören zu einer Medaille, zu einer Person, die insgesamt gefördert und entwikkelt (»gesellschaftsfähig « gemacht) werden muss:

- persönlich im Sinne von Erziehung, Beratung und Therapie, im Sinne der Förderung der Persönlichkeit mit dem Ziel der sozialen Eingliederung und

- beruflich im Sinne von Qualifizierung, Vermittlung und Beschäftigung mit dem Ziel Wissen und Fähigkeiten zu vermitteln und die berufliche Eingliederung zu erreichen.
Dies ist das besondere Profil und die besondere Stärke der Jugendsozialarbeit und gleichzeitig die Entsprechung dieses ganzheitlichen Anspruchs, der im Paragraf 13 des Kinder- und Jugendhilfegesetzes formuliert und grundsätzlich angelegt ist. Auch hier wieder ein eindeutiger Hinweis darauf, dass die Basis und der Ausgangspunkt der Jugendsozialarbeit eindeutig in der Jugendhilfe liegen, auch wenn die Realität der Finanzierung der Einrichtungen inzwischen in andere Richtungen zu weisen scheint. Trotzdem bleiben natürlich Fragen offen:

- Wo ist denn innerhalb dieses breiten inhaltlichen Spektrums - Therapie, Beratung, Erziehung, Bildung, Qualifizierung, Beschäftigung und Vermittlung das inhaltliche Zentrum?

- Wo schlägt das Herz der Jugendsozialarbeit? Ein ständiges Sowohl-als-auch kann ja auch ganz schön anstrengend sein, vor allem für die Mitarbeiterinnen und Mitarbeiter.

- Wofür sind wir denn eigentlich zuständig bei diesen vielen Aufgaben?

- Was können wir überhaupt leisten und wofür sind wir im Grunde ausgebildet?

Diese Fragen führen zum Verständnis von Bildung und der Position, die Bildung auf der inhaltlichen Skala und damit innerhalb der Jugendsozialarbeit einnehmen soll. Meine Behauptung vorweg: Bildung soll eine zentrale, eine integrative Position einnehmen. Und: Bildung ist mehr als das, was bisher in den grundsätzlichen Überlegungen der Jugendsozialarbeit formuliert worden ist. Aus dieser Behauptung leite ich meine Botschaft ab, die vor dem Hintergrund meiner Annäherungsversuche im Mittelpunkt und zur Diskussion stehen soll:

Die Jugendsozialarbeit besteht aus Einrichtungen der Jugendhilfe, die aber als zentrale Dienstleistung Bildung im eigentlichen Sinne anbieten. Die Jugendsozialarbeit kann für sich auf diese Weise einen eigenständigen Bildungsauftrag in Anspruch nehmen und Bildung kann so flankiert von Erziehung und Qualifizierung - als das identitätsstiftende Zentrum der Jugendsozialarbeit, sozusagen als konzeptionelle Schnittstelle bezeichnet werden. Ich will versuchen, Bildung in diesem Zusammenhang als Ziel von Erziehung und Qualifizierung zu fassen und gleichzeitig als eigenständige Methode zu begreifen, die auf beidem, auf unseren Er- ziehungs- und unseren Qualifizierungsbemühungen aufbaut.

Erziehung wird in der pädagogischen Literatur ziemlich übereinstimmend als der beabsichtigte Versuch bezeichnet, die Persönlichkeit der zu Erziehenden zu fördern und im positiven Sinne zu entwickeln, also ganz im Sinne unserer bisherigen Bestimmungsversuche für die Jugendsozialarbeit. Aber: Was heißt positiv? Da wird klar, wie wichtig die Frage nach den Zielen von Erziehung ist, denn Erziehung besteht immer aus der sehr ambivalenten, uneindeutigen und konfliktreichen Doppelaufgabe der Sozialisation und der Personalisation. Also aus dem Versuch, gleichzeitig

- bestimmte Normen, Werte und Regeln, die in unserer Gesellschaft gelten zu vermitteln (Sozialisation) und

- zur Selbstständigkeit, zur Individualität, zur immer größer werdenden Unabhängigkeit der Jugendlichen beizutragen (Personalisation).

Das heißt, Erziehung hat die Aufgabe, einerseits in die Gesellschaft einzuführen, in der wir ja nun mal alle leben und andererseits gegen sie, vor allem gegen ihre Risiken und Nebenwirkungen, zu »immunisieren «. Risiken und Nebenwirkungen übrigens, die ja in der Jugendhilfe häufig und zu Recht als Ursache dafür verantwortlich gemacht werden, dass sich Jugendliche überhaupt in den Einrichtungen der Jugendhilfe befinden. Für diese schwierige Aufgabe, Regeln beibringen und gleichzeitig selbstständig machen, ist eine Beziehung notwendig. Ohne Beziehung keine Erziehung, und: Diese Beziehung ist immer ungleichgewichtig, nie ein reines Partnerschaftsverhältnis, immer von einem Machtgefälle, einem Autoritätsverhältnis geprägt. Erziehung baut auf diesem Machtunterschied auf und muss gleichzeitig versuchen, ihn so schnell wie möglich wieder abzubauen, die Jugendlichen in die Selbstständigkeit und Unabhängigkeit zu entlassen. Trotzdem muss diese Ausübung von Macht, die notwendig ist, begründet und legitimiert werden, gerade weil sie nicht absolut und beliebig sein soll und nicht in Gewalt ausarten darf, sondern nur als befristeter Auftrag erteilt worden ist und auch so verstanden werden soll. Das ist ein entscheidender Punkt, gerade in der Jugendhilfe, nämlich: Dass die notwendige Ausübung von Macht und Autorität nicht willkürlich sein soll und nicht in bestimmte Formen von Gewalt ausarten darf, so wie es die 
meisten Jugendlichen schon zur Genüge erlebt haben und vielleicht gerade deswegen dieser Hilfen bedürfen. Die notwendige Ausübung von Macht und Autorität muss als zeitlich befristeter und klar für alle nachvollziehbar begründeter Auftrag verstanden werden.

Und dafür kann Bildung als Ziel von Erziehung eine wichtige Begründung sein: Das zeitlich befristete Mandat zur Ausübung von Macht und Autorität (nicht von Gewalt!), das dadurch legitimiert wird, dass Bildung und durch sie gesellschaftliche Mündigkeit und Teilhabe, Selbstbestimmung und Selbstverantwortung der Jugendlichen möglich werden. Was das heißt, will ich gleich näher erklären, vorher aber noch zur zweiten Voraussetzung.

Qualifizierung ist die Grundlage von Bildung, auch ohne Qualifizierung ist Bildung nicht möglich. Wörtlich übersetzt heißt Qualifizierung Befähigung, Eignung und auch Berechtigung; die Berechtigung dabei zu sein in dieser Gesellschaft, könnten wir in diesem Zusammenhang sagen. Konkret bedeutet dies zunächst: Dazulernen, Fakten wissen, sich Kompetenzen aneignen, Kenntnisse und Fertigkeiten erlangen. Das ist Qualifizierung und damit die zentrale und notwendige Grundlage für berufliche und soziale Integration, ohne die es nicht geht. Aber Qualifizierung ist eben auch nur eine Grundlage und sie ist es auch nur dann, wenn diese Kompetenzen, Kenntnisse und Fertigkeiten geeignet, verwertbar, marktgerecht und aktuell sind, an der Lebens- und Arbeitswelt der Jugendlichen, um deren Integration es geht, orientiert sind. Diese Grundlage »Qualifizierung « darf nun aber nicht gleich gesetzt werden mit Bildung. Dies scheint ein großes und zentrales Missverständnis zu sein: Bildung ist viel mehr als lernen, wissen und können. Georg Kerschensteiner, ein Begründer der Berufspädagogik hat dieses Verständnis vor fast hundert Jahren auf den Punkt gebracht und zugespitzt formuliert: »Bildung ist das, was übrig bleibt, wenn alles Gelernte wieder vergessen ist. « Bildung fängt also erst so richtig an, wenn wir schon etwas gelernt haben, also schon qualifiziert sind.

Und damit sind wir am entscheidenden Punkt angelangt, nämlich dort, worauf Erziehung und Qualifizierung abzielen, nämlich auf Bildung. Aber nur unter der Bedingung, dass wir jetzt Bildung anders

\section{»ugendsozialarbeit ist Bildung «}

Jugendsozialarbeit hat neben der Aufgabe zu qualifizieren und Wissen zu vermitteln auch den Auftrag zu bilden:

These 1: Bildung findet im Alltag statt, also auch im Alltagsgeschäft der Jugendsozialarbeit, in der Werkstatt, in Beratungssituationen und in erlebnispädagogischen Maßnahmen. Dies ist keineswegs konsensfähig. Bildung wird oft zu Unrecht als etwas Elitäres, Besseres, Höherstehenderes begriffen, das Besserverdienenden, den Bildungsbürgerinnen und Bildungsbürgern vorbehalten zu sein scheint. Bildung machen die Lehrerinnen und Lehrer in Gymnasien, die Professorinnen und Professoren an den Universitäten, während die Sozialpädagoginnen und Sozialpädagogen für die Erziehung und die Anleiterinnen und Anleiter für die Ausbildung zuständig sind - ein kleiner feiner Unterschied, der in den letzten 150 Jahren wesentlich zur Erhaltung unserer Schichtgesellschaft beigetragen hat und tief in unseren Köpfen verwurzelt ist.

These 2: Bildung ist nichts Passives, was mit einem passiert, sondern Bildung ist für alle Beteiligten ein aktiver Prozess, also für die, die Bildung machen und für die, die sich bilden wollen und gebildet werden sollen. Auch diese These ist nicht verständlich. $\mathrm{Zu}$ oft erleben wir in Schulen, auf Seminaren oder Kursen Bildung als etwas, dass es zu schlucken und anschließend bei Prüfungen unbearbeitet wiederzugeben gilt. Bildung als etwas, was mit passiver Aufnahme und Wiedergabe zu tun hat.

These 3: Bildung ist immer politisch, Bildung ist immer auch auf die Gesellschaft im Großen und im Kleinen bezogen, also nie nur auf Inhalte und Sachverhalte, die es zu vermitteln gilt. Auch dies ist nicht selbstverständlich. Nicht nur Roman Herzog hat von Bildung als einem Rohstoff geredet, den es in möglichst großen Mengen zu produzieren gilt, zur Sicherung des wirtschaftlichen Standorts Deutschland. Das sitzt tief in den Köpfen und erst Johannes Rau hat kurz vor seinem Tod versucht, die Sache zu relativieren, indem er mehr Teilhabe und Chancengleichheit für alle im Hinblick auf Bildung und Bildungschancen gefordert hat.

Joachim König

verstehen, als es in der Arbeit der Bildungsträger oft der Fall ist und als es wohl auch in unseren Köpfen und Konzepten wahrscheinlich noch Realität ist. Meine Botschaft noch einmal: Jugendsozialarbeit ist Teil der Jugendhilfe mit einem klaren Erziehungsauftrag für eine bestimmte Zielgruppe. Dieses pädagogische Mandat ist ihr Ausgangspunkt, ihre Basis. Aber sie hat deshalb und auch darauf aufbauend einen Bildungsauftrag, der über die Qualifizierung und Wissensvermittlung bei den Bildungsträgern weit hinausgeht. Dieser über 200 Jahre alte, humanistische Bildungsbegriff der Aufklärung könnte - mein Vorschlag - für die Jugendsozialarbeit eine Leitidee, ein Identifikationszentrum abgeben, in Abgrenzung zu dem, was heute oft missverständlich unter Bildung verstanden wird. Dieser Bildungsbegriff kann konkretisiert und auf die Praxis der Jugendsozialarbeit angewandt werden (vgl. Kasten auf Seite 48: "Jugendsozialarbeit ist Bildung «).

Der Bildungsbegriff selbst kann in Form eines Setzkastens dargestellt werden, eines Setzkastens, der aus mehreren Feldern und aus Einzelteilen besteht, die alle miteinander eine gemeinsame Bedeutung bekommen, aber auch für sich selbst genommen wichtige Einzelfunktionen übernehmen und in je unterschiedlicher Zusammensetzung konkretisierbar sind. Dieser Setzkasten besteht aus acht Feldern, die ich in drei Schritten der Reihe nach erklären möchte (vgl. Abb. 3).

Schritt 1: Bildung hat immer eine individuelle und eine politische Seite, es geht also immer um die eigene Person und auch um das »Drumherum «, um die Gesellschaft, in der Jugendsozialarbeit gemacht wird, in der Steuern gezahlt und nach einem bestimmten System wieder ausgegeben werden. Um die Stadt, in der in manchen Teilen Probleme existieren und in manchen eben nicht. Bildung ist also nie nur auf einzelne Menschen bezogen, die sich bilden oder gebildet werden, die Systemfrage entscheidet immer mit. Dies ist ein wichtiges Regulativ, vor allem für die Pädagogenköpfe und -herzen, es hilft, das »Überindividualisieren « $\mathrm{zu}$ vermeiden.

Schritt 2: Bildung hat immer etwas mit Rechten und mit Pflichten zu tun. Deshalb immer auch etwas mit Verantwortung. Verantwortung mir selbst gegenüber und meinem Umfeld gegenüber, der Gesellschaft, dem Stadtteil oder dem Team in einer Werkstatt gegenüber. Das 
heißt: Zum Recht auf Selbstbestimmung und Mitbestimmung gehört immer die Pflicht zur Selbstverantwortung und Mitverantwortung.

Schritt 3: Bildung hat immer eine pädagogische Seite, die auf die Jugendlichen zielt, für die wir uns pädagogisch verantwortlich fühlen und Bildung hat immer eine politische Seite, die die Hintergründe und Bedingungen dieses pädagogischen Verhältnisses und damit auch uns selber werden und Verantwortung konkret zu übernehmen, für sich selbst und für die soziale Umwelt. Wiederum das Recht sich einzumischen und die Pflicht Verantwortung zu übernehmen

- und Bildung meint immer auch, sich für Bedingungen, dass all dies möglich ist, einzusetzen

Spätestens hier geht es nicht mehr nur um die Jugendlichen, sondern auch um uns als Verantwortliche, und es geht bei

\section{"Jugendsozialarbeit verfolgt sieben Ansätze: Therapie, Beratung, Erziehung, Bildung, Qualifizierung, Beschäftigung, Vermittlung “}

als Verantwortliche in der Jugendsozialarbeit betrifft. Eine pädagogische Seite, die lernen, beibringen, Wissen erwerben und Meinung bilden, motivieren bedeutet und eine politische Seite, die die Strukturen und Bedingungen thematisiert unter denen gelernt und motiviert wird, auch die Berechtigungen, wer denn in diesem Land was lernen darf und was nicht.

Das heißt, es ist zwar wichtig, und auch Teil von Bildung, bestimmte Dinge überhaupt zu können, gelernt zu haben und in die Tat umzusetzen, aber das ist eben nicht alles. Es ist genauso wichtig, dass wir dafür sorgen, dass Selbstbestimmung, Selbstverantwortung, Mitbestimmung und Mitverantwortung gewollt sind, den Jugendlichen erlaubt werden, und dass die Bedingungen dafür existieren, dass sie es umsetzen können, dass nicht alles Lippenbekenntnis und Sonntagsrede bleibt. Dies ist unsere Verantwortung, die Seite von Bildung, die uns als Mitarbeiter und Mitarbeiterinnen betrifft und von uns erwartet, dass wir aktiv werden und aus dem Schatten unseres rein pädagogischen Blicks heraustreten.

Zusammengefasst lässt sich also sagen: Bildung meint unsere Bemühungen um vier Dinge:

- das Wissen und Können der Jugendlichen, ihr Recht darauf und die Pflicht sich darum zu bemühen

- die Fähigkeit der Jugendlichen, dieses Wissen bewerten zu können, eine eigene Meinung dazu zu erarbeiten, das Recht auf eine eigene Meinung und die Pflicht, sich darum zu bemühen

- auf der Basis dieser Meinung aktiv zu
Bildung weit über das pädagogische »1:1-Geschäft « hinaus. Dieser Bildungsbegriff passt deshalb so gut zu den Grundsätzen des Kinder- und Jugendhilfegesetzes, besonders zum Paragraf 13, weil er eben nicht nur einen Lernbedarf anmeldet, sondern darüber hinaus auch Motivations- und Handlungsbedarfe weckt. Letztlich, wenn auch im Kleinen, in einem sehr politischen Sinne - bezogen auf die Jugendlichen und bezogen auf uns als Verantwortliche. Darin besteht der Bildungsauftrag der Jugendsozialarbeit.

Die einzelnen Felder des Setzkastens können nun konkretisiert, ausgefüllt werden, mit einzelnen Maßnahmen, Methoden, Angeboten und Inhalten, die eine Rolle, beispielsweise im Alltagsgeschäft der berufsbezogenen Jugendhilfe, jeweils für sich und insgesamt miteinander, im Hinblick auf einen solchen Bildungsbegriff spielen, etwa

- während der Lerntage, wenn es um die Vermittlung von Grund- und Allgemeinwissen geht,

- auf den Sitzungen der Personalvertretungen mit den Jugendlichen, wo diskutiert und Meinungsbildung betrieben wird,

- während der Arbeiten im »Hausservice«, wo reihum alle Jugendlichen Dienstleistungen für die Gemeinschaft (putzen, aufräumen, Brotzeit holen) verrichten müssen und so Mitverantwortung lernen und

- in Projekten im Stadtteil, wo öffentliche Meinung vertreten und mit gebildet wird.
Zusammenfassend lässt sich sagen: Die Einrichtungen der Jugendsozialarbeit sind Träger der Jugendhilfe. Dies ist ihre konzeptionelle Basis. Durch den zentralen Gedanken, die Persönlichkeit der Jugendlichen zu fördern und zu entwickeln, indem wir die soziale und berufliche Eingliederung in diese Gesellschaft versuchen, Erziehung und Qualifizierung konkret unternehmen, können wir aber für uns in Anspruch nehmen, dass eine unserer zentralen Dienstleistungen Bildung für die Jugendlichen ist und, dass wir so definiert einen eigenständigen Bildungsauftrag für benachteiligte Jugendliche erfüllen und zwar einen Bildungsauftrag in einem sehr umfassenden Sinne:

- pädagogisch und politisch bildend, nicht nur beruflich qualifizierend

- ganzheitlich fördernd, nicht nur auf die Qualifizierung von Wissen und Können bezogen

- langfristig begleitend, nicht nur modul-, maßnahmen- und interventionsbezogen

- politisch engagiert, nicht nur dienstleistungsorientiert

- das Recht auf Bildung, aber auch die Pflicht dazu betonend

- und nahe an der sozialen Wirklichkeit und nicht nur an Lehrplänen und Curricula orientiert.

\section{5.}

Bildung als Methode und gleichzeitig als Ziel der Jugendsozialarbeit könnte also heißen:

- den Jugendlichen etwas beizubringen, ihnen zu zeigen, wie die Welt funktioniert,

- ihnen zu erklären, wie wichtig dabei eine eigene Meinung ist

- sie immer wieder dazu zu ermuntern, aktiv zu werden in der Verantwortung für sich und für die Welt um sie herum

- ihnen klarzumachen, dass das Ganze an Bedingungen geknüpft ist, für die sie sich und wir uns miteinander einsetzen müssen, und deshalb

- diese Rechte, die sie haben, immer auch mit Pflichten verbunden sind, die wir alle, also auch sie immer wieder übernehmen müssen.

Wenn wir an diesen Punkten in unserem Alltagsgeschäft immer wieder in kleinen Schritten vorankommen, dann haben wir viel geschafft, im Sinne der Jugendlichen, für die wir Verantwortung tragen. 